EXTENDED REPORT

\title{
Enhanced endothelium derived hyperpolarising factor activity in resistance arteries from normal pressure glaucoma patients: implications for vascular function in the eye
}

\author{
C Cleary, C H Buckley, E Henry, P McLoughlin, C O'Brien, P W F Hadoke
}

Br J Ophthalmol 2005;89:223-228. doi: 10.1136/bjo.2004.044446

See end of article for authors' affiliations

Correspondence to: Dr Patrick W F Hadoke, School of Molecular and Clinical Medicine, Endocrinology Unit, 2nd Floor OPD, Western General Hospital, Crewe Road, Edinburgh EH4 2XU, UK; phadoke@ staffmail.ed.ac.uk

Accepted for publication 22 July 2004

\begin{abstract}
Background/aims: Endothelial cell dysfunction in the ocular circulation may contribute to normal pressure glaucoma (NPG). This study aimed to investigate the contributions made by endothelium derived relaxing factors to relaxation of (1) subcutaneous resistance arteries from patients with NPG, and (2) porcine ciliary arteries.

Methods: Human gluteal resistance arteries were isolated from seven patients with NPG and matched controls. Human and porcine arteries produced endothelium dependent relaxation when exposed to acetylcholine (ACh) $\left(10^{-9}-3 \times 10^{-5} \mathrm{M}\right)$ or bradykinin $\left(10^{-10}-3 \times 10^{-6} \mathrm{M}\right)$. Pharmacological agents were used to inhibit the nitric oxide pathway (L-arginine analogues, soluble guanylate cyclase inhibitor), endothelium derived hyperpolarising factor (EDHF) activity (potassium channel antagonists), and prostaglandin synthesis (cyclo-oxygenase inhibitors).

Results: In all arteries, endothelium dependent relaxation was attenuated by nitric oxide (NO) inhibition or potassium channel blockade, but not by cyclo-oxygenase inhibition. Inhibition of ACh mediated relaxation by potassium channel antagonists was greater $(p<0.05)$ in patients with NPG (Emax, 55.4\% (SD 8.16\%) relaxation, $n=4)$ than controls (Emax, $81.8 \%(6.0 \%), n=5)$. In contrast, combined inhibition of NO synthase (NOS) and cyclo-oxygenase produced similar inhibition of ACh mediated relaxation in both groups.

Conclusions: The enhanced contribution of EDHF to ACh mediated relaxation in systemic resistance arteries from NPG patients may contribute to the maintained endothelium mediated relaxation in these vessels. EDHF also contributes significantly to bradykinin mediated relaxation in porcine ocular ciliary arteries. Therefore, similar changes in the balance of relaxing factors in the ocular circulation could influence the response of the eye to vascular endothelial dysfunction in NPG.
\end{abstract}

ntraocular pressure is not elevated in approximately one third of all cases of glaucoma, leading to a condition described as normal pressure glaucoma (NPG). ${ }^{1}$ As glaucoma, particularly NPG, is often accompanied by widespread cerebrovascular and systemic cardiovascular disease, ${ }^{2}$ it has been proposed that vascular dysfunction is a contributory factor in the pathogenesis of this condition. ${ }^{3}$ Indeed, NPG may be one component of a generalised vascular defect that produces alterations in both the ocular and systemic circulations. Certainly, patients with NPG have increased resistance and reduced blood flow velocity within the ophthalmic circulation ${ }^{4-6}$ while systemic vascular dysfunction is indicated by the high prevalence of migraine ${ }^{7}$ in these individuals. Direct evidence of systemic vascular dysfunction is provided by the association of NPG with reduced blood flow in the fingers, ${ }^{8}$ impaired nitric oxide (NO) mediated, endothelium dependent relaxation in the forearm circulation, ${ }^{9}$ and enhanced contraction in systemic resistance arteries. ${ }^{10}$

Intriguingly, our previous in vitro investigation of resistance artery function, using wire myography, found endothelium dependent relaxation to be unimpaired ${ }^{10}$ in patients with NPG. This may, however, be related to the fact that relaxation of small resistance arteries is mediated by the release of both $\mathrm{NO}$ and an, as yet unidentified, endothelium derived hyperpolarising factor (EDHF). ${ }^{11}{ }^{12}$ In some conditions, including hypercholesterolaemia, ${ }^{13}{ }^{14}$ impairment of the
NO component of relaxation may be balanced by an increase in EDHF activity. Similar mechanisms may be significant in the eye as EDHF also contributes to regulation of tone in ocular arteries. ${ }^{15}$

Thus, we postulated that endothelium dependent relaxation may be maintained in systemic resistance arteries from patients with glaucoma by an alteration in the balance between EDHF and NO. The specific aims of the investigation were to determine ( 1 ) the effect of NPG on the contributions of NO and EDHF to acetylcholine (ACh) mediated relaxation of human resistance arteries and (2) the contribution made by EDHF to endothelium dependent relaxation in ocular blood vessels.

\section{MATERIALS AND METHODS}

\section{Tissues}

Human subcutaneous resistance arteries

Arteries were obtained from seven patients with newly diagnosed NPG, and matched controls (table 1). Patients with NPG had an intraocular pressure less than $22 \mathrm{~mm} \mathrm{Hg}$ on diurnal phasing (with Goldmann applanation tonometry), open anterior chamber angles on gonioscopy, optic disc cupping (with a cup:disc ratio $>0.6$ ), and either thinning or

Abbreviations: ACh, acetylcholine; EDHF, endothelium derived hyperpolarising factor; NO, nitric oxide; NOS, nitric oxide synthase; NPG, normal pressure glaucoma 
notching of the neural rim, and glaucomatous field loss (arcuate scotoma, nasal step, paracentral defect) on the Humphrey perimeter (Humphrey Instruments, USA) using the 24-2 threshold program (average mean deviation $-7.29 \mathrm{~dB}$ and corrected pattern standard deviation $9.92 \mathrm{~dB}$ ). Radiology was carried out when indicated to exclude intracranial causes of optic disc anomalies or visual field loss. Controls (recruited from friends and relatives of participants and by advertisement) had a normal ocular examination, intraocular pressure within normal limits, and intact visual fields on automated analysis. Patients were either untreated $(n=3)$ or were being treated $(n=4)$ with $\beta$ blockers ( $0.5 \%$ betaxolol hydrochloride (Betoptic), Alcon Ltd, UK), alone $(\mathrm{n}=3)$ or in combination with a carbonic anhydrase inhibitor $(2 \%$ dorzolamide hydrochloride (Trusopt), Merck Sharp \& Dohme, UK; $\mathrm{n}=1$ ). The four patients receiving treatment did not take their medication on the day of biopsy. None of the subjects were receiving additional systemic vasoactive drugs and all were nonsmokers. Venous blood was obtained for measurement of plasma cholesterol. The study protocol was approved by Lothian research ethics committee and conformed to the Declaration of Helsinki. All subjects gave written informed consent before enrolment in the study.

Subcutaneous resistance arteries were obtained from a biopsy of skin and subcutaneous fat $(2 \mathrm{~cm} \operatorname{long} \times$ $1 \mathrm{~cm} \times \mathrm{lcm}$ ) taken from the gluteal region under local anaesthetic (Xylocaine; 2\% lidocaine hydrochloride with adrenaline, Astra Pharmaceuticals, UK).

\section{Porcine posterior ciliary arteries}

Porcine (male and female; large white/Landrace; age 5 months) eyes were obtained from a local abattoir immediately following slaughter, enucleated, and placed in ice cold HEPES buffer ( $130 \mathrm{mM} \mathrm{NaCl}, 5.6 \mathrm{mM} \mathrm{KCl}, 1 \mathrm{mM} \mathrm{MgCl}_{2}$, $10 \mathrm{mM}$ HEPES, and $1.1 \mathrm{mM}$ glucose; $\mathrm{pH} 7.35)$. Eyes were stored at $4^{\circ} \mathrm{C}$ and used over 3 successive days with posterior ciliary arteries isolated on the morning of each experiment. Only one eye from each animal was used.

\section{Functional analysis}

Isometric function of human subcutaneous resistance arteries and porcine ciliary arteries was measured using standard small vessel myography. ${ }^{16}$ Briefly, arteries were mounted as ring preparations on two $40 \mu \mathrm{m}$ intraluminal wires in a chamber containing either (i) physiological salt solution (PSS; composition in mM: $\mathrm{NaCl} 119, \mathrm{KCl} 4.7, \mathrm{MgSO}_{4}$ 1.17, $\mathrm{KH}_{2} \mathrm{PO}_{4}$ 1.18, D glucose 5.5, $\mathrm{K}_{2}$ EDTA 0.026, $\mathrm{NaHCO}_{3}$ $\left.25, \mathrm{CaCl}_{2} 2.5\right)$ for subcutaneous resistance arteries or (ii) Krebs-Henseleit solution (composition $123 \mathrm{mM} \mathrm{NaCl}$, $5.4 \mathrm{mM} \mathrm{KCl}, 2.4 \mathrm{mM} \quad \mathrm{CaCl}_{2}, 0.8 \mathrm{mM} \mathrm{MgSO}_{4}, 20 \mathrm{mM}$ $\mathrm{NaHCO}_{3}, 0.9 \mathrm{mM} \mathrm{NaH} \mathrm{PO}_{4}$ and $5.5 \mathrm{mM}$ glucose) for porcine ciliary arteries. The solutions were maintained at $37^{\circ} \mathrm{C}$ and gassed with $95 \% \quad \mathrm{O}_{2}, 5 \% \mathrm{CO}_{2}$. Following a 30 minute equilibration period, the resting tension internal circumference relationship was determined for each artery by stepwise stretching of the vessel and application of the LaPlace equation, as previously described. ${ }^{16}$ The internal circumference $\left(\mathrm{L}_{100}\right)$ of each artery under a transmural pressure of $100 \mathrm{~mm} \mathrm{Hg}(13.3 \mathrm{kPa})$ was calculated and the vessel was stretched to its optimum resting setting $\left(0.9 \mathrm{~L}_{100}\right){ }^{17}$

\section{Experimental protocols}

\section{Human subcutaneous resistance arteries}

Following a standard start procedure, ${ }^{10}$ cumulative concentration response curves were obtained with noradrenaline (NA) $\left(10^{-9}-3 \times 10^{-5} \mathrm{M}\right)$ and, following pre-contraction with NA $\left(10^{-7}-10^{-6} \mathrm{M}\right)$, to ACh $\left(10^{-9}-3 \times 10^{-5} \mathrm{M}\right)$ in each arterial ring. The concentration response curve for ACh was then repeated in the presence of one of the following combinations of inhibitors:

(a) $\mathrm{N}^{\mathrm{G}}$-nitro-L-arginine (L-NOARG; $10^{-4} \mathrm{M}$ ) and indomethacin (Indo; $10^{-5} \mathrm{M}$ ), for 45 minutes, to inhibit nitric oxide (NO) synthase and cyclo-oxygenase, respectively.

(b) $1 \mathrm{H}-[1,2,4]$ oxadiazolol [4,3-a]quinoxalin-1-one (ODQ; $\left.10^{-5} \mathrm{M}\right)$, for 10 minutes, to inhibit soluble guanylate cyclase.

(c) Charybdotoxin (ChTx; $\left.5 \times 10^{-8} \mathrm{M}\right)$ and apamin (Apa; $3 \times 10^{-8} \mathrm{M}$ ), for 10 minutes, to inhibit the effects of endothelium derived hyperpolarising factor.

(d) A combination of L-NOARG, Indo, ChTx, and Apa.

Each artery was exposed in random order to between one and four of the combinations given.

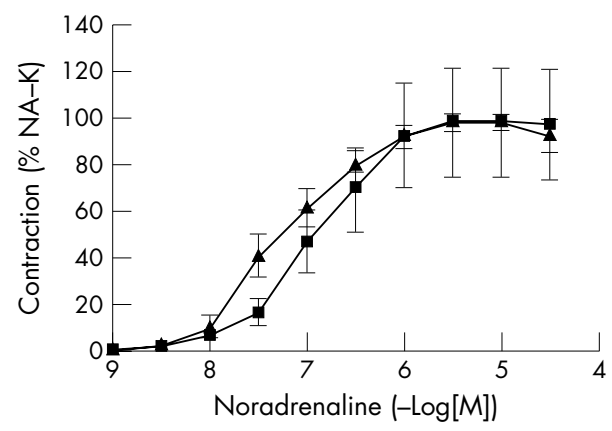

Figure 1 Concentration-response curves to noradrenaline in subcutaneous resistance arteries from patients with normal pressure glaucoma (triangles, $n=7$ ) or from matched controls (squares, $n=7$ ). Each point represents mean (SEM). NA-K, high (125 mM) potassium PSS containing $10^{-5} \mathrm{M}$ noradrenaline.

Table 1 Characteristics of control subjects and patients with normal pressure glaucoma (NPG)

\begin{tabular}{llll}
\hline & Control & NPG & p Value \\
\hline Sex (M:F) & $4: 3$ & $4: 3$ & - \\
Age (years) & $53(2)$ & $63(3)$ & NS (0.09) \\
IOP (mm Hg) & $15.5(1.2)$ & $16.2(1.1)$ & NS (0.67) \\
Mean arterial blood pressure & $82.6(2.5)$ & $82.2(3.4)$ & NS (0.44) \\
(mm Hg) & & & NS $(0.29)$ \\
Heart rate (bpm) & $70.8(4.2)$ & $63.0(3.7)$ & NS $(0.53)$ \\
Weight (kg) & $65.8(2.7)$ & $74.6(6.5)$ & NS $(0.17)$ \\
Cholesterol (mmol/l) & $5.40(0.44)$ & $4.47(0.45)$ & \\
\hline
\end{tabular}

Values are expressed as mean (SEM); $n=6-7$. Statistical comparisons were made using Student's unpaired $t$ test IOP, intraocular pressure; bpm, beats per minute; NS, not significant. 


\section{Porcine ciliary arteries}

Two arterial rings from each eye were assessed in parallel. Following a standard start, the rings were constricted with a submaximal concentration $\left(\sim \mathrm{EC}_{70}\right)$ of prostaglandin $\mathrm{F}_{2 \alpha}$ $\left(\mathrm{PGF}_{2 \alpha}\right)$ and cumulative concentration-response curves generated using bradykinin (BK; $1 \times 10^{-10}$ to $3 \times 10^{-6} \mathrm{M}$ ). Relaxation greater than $60 \%$ was taken to indicate a functional endothelium.

Concentration-response curves to BK were then repeated following incubation (30 minutes) with:

(a) $\mathrm{L}-\mathrm{N}^{\mathrm{G}}$-nitro-arginine methyl ester (L-NAME; $10^{-3} \mathrm{M}$ ), to inhibit NO synthase. Controls were exposed to vehicle.

(b) L-NAME $\left(10^{-3} \mathrm{M}\right)$ plus meclofenamic acid $\left(10^{-5} \mathrm{M}\right)$, to inhibit cyclo-oxygenase. Controls were exposed to L-NAME alone, or

(c) L-NAME $\left(10^{-3} \mathrm{M}\right)$, plus meclofenamic acid $\left(10^{-5} \mathrm{M}\right)$ with ChTx $\left(1 \times 10^{-7} \mathrm{M}\right)$ and Apa $\left(1 \times 10^{-7} \mathrm{M}\right)$, to inhibit EDHF. Controls were exposed to L-NAME and meclofenamic acid.

\section{Drugs}

Salts were obtained from BDH (Poole, Dorset, UK). Noradrenaline bitartrate and acetylcholine chloride were from Sigma (Dorset, UK). $\mathrm{N}^{\mathrm{G}}$-nitro-L-arginine, ODQ (1H-[1,2,4] oxadiazole[4,3-a]quinoxalin-1-one), charybdotoxin, and apamin were obtained from Alexis Biochemicals (Nottingham, UK). Drugs were dissolved in deionised water, except indomethacin which was dissolved in $1.5 \times 10^{-3} \mathrm{M}$ $\mathrm{Na}_{2} \mathrm{CO}_{3}$ (final bath concentration of $\mathrm{Na}_{2} \mathrm{CO}_{3}$ did not exceed $0.015 \mathrm{mM}$ ), and stored in aliquots at $-20^{\circ} \mathrm{C}$.

\section{Statistical analysis}

Relaxation responses are expressed as a percentage of the induced pre-contraction to NA or $\mathrm{PGF}_{2 \alpha}$. For each concentration-response curve the molar concentration required to produce $50 \%$ of the maximum response $\left(\mathrm{EC}_{50}\right.$ for $\mathrm{NA}, \mathrm{IC}_{50}$ for ACh) was calculated by fitting the Hill equation (Fig P, Biosoft, UK); sensitivities were expressed as $\mathrm{pD}_{2}$ and -log $\mathrm{IC}_{50}$, respectively. All results are given as the mean (SEM) for $\mathrm{n}$ experiments, where $\mathrm{n}$ represents the number of subjects. Maximum response, $\mathrm{pD}_{2}$ and $-\log \mathrm{IC}_{50}$ values were compared using Student's unpaired $t$ test and differences considered significant when $\mathrm{p} \leqslant 0.05$. Entire curves were compared by analysis of variance with the Bonferroni post hoc test.

\section{RESULTS}

\section{Human subcutaneous resistance arteries}

There were no significant differences in the demographics between patient and control groups, in particular no differences in mean arterial blood pressure or cholesterol (table 1). Eighteen viable vessels were isolated from the seven controls and 22 from the seven (NPG) patients. The internal diameter of resistance arteries isolated from gluteal biopsies used in this study was similar $(\mathrm{p}=0.60)$ in patients with glaucoma (226 (11) $\mu \mathrm{m})$ and control subjects (218 (13) $\mu \mathrm{m})$. The contractile responses evoked by a combination of NA $\left(10^{-5} \mathrm{M}\right)$ with $125 \mathrm{mM}$ potassium (NA-K) were not significantly different between the two groups (patients 3.15 (0.36) $\mathrm{mN} / \mathrm{mm}$; controls 2.57 (0.32) $\mathrm{mN} / \mathrm{mm}, \mathrm{p}=0.25)$.

NA mediated contraction (fig 1 ) was similar in arteries from patients (Emax, $3.12(0.37) \mathrm{mN} / \mathrm{mm} ; \mathrm{pD}_{2}, 7.28$ (0.18; $\mathrm{n}=7)$ and controls (2.23 (0.31) $\mathrm{mN} / \mathrm{mm}, \mathrm{p}=0.09 ; 6.89$ $(0.10 ; \mathrm{p}=0.08 ; \mathrm{n}=7)$, similar to our previous findings. ${ }^{10}$ In patients with NPG, arterial sensitivity to ACh was greater than controls but the maximum relaxation was similar in the two groups (fig 2A; table 2). In accordance with previous investigations, ${ }^{11}$ the combination of LNOARG/Indo/ChTx/ apamin virtually abolished relaxation in arteries from both groups (fig 2A; table 2). Exposure to L-NOARG with Indo (fig 2B), or to ODQ (fig 2C), reduced the magnitude of ACh mediated relaxation to a similar extent ( $\sim 20-30 \%$ inhibition; $\mathrm{p}=0.0008$ and $\mathrm{p}=0.002$, respectively) in arteries from patients and controls (table 2). In contrast, exposure to ChTx/apamin (fig 2D) produced greater inhibition of relaxation $(p<0.0001)$ in arteries from patients with NPG than in those from controls (table 2).

\section{Porcine ciliary arteries}

Following submaximal contraction with $\mathrm{PGF}_{2 \alpha}$, posterior ciliary arteries produced a concentration dependent relaxation to BK before exposure to inhibitors (table 3).

Exposure of ciliary arteries to L-NAME attenuated, but did not abolish, BK mediated relaxation (fig 3A; table 3). Addition of meclofenamic acid in the presence of L-NAME, did not further attenuate BK mediated relaxation (fig 3B; table 3 ). The combination of charybdotoxin and apamin abolished $(\mathrm{p}<0.0002)$ the L-NAME insensitive component of the $\mathrm{BK}$ mediated contraction (fig 3C; table 3).

\section{DISCUSSION}

This study, the first to assess EDHF activity in NPG, has demonstrated that the contribution made by EDHF to ACh mediated relaxation is enhanced in resistance arteries from patients with this condition. This enhancement could not be attributed to hypercholesterolaemia or elevated blood pressure and suggests ACh mediated relaxation is conserved in these arteries by an alteration in the balance of endothelium derived relaxing factors. The confirmation that both EDHF and NO contribute to endothelium dependent relaxation of porcine ciliary artery suggests that similar changes could influence the effect of disease on blood flow in the ocular circulation.

The contribution of EDHF to endothelium dependent relaxation increases as vessel size decreases, ${ }^{18}$ with EDHF having a significant role in relaxation of human ${ }^{11}$ and rodent $^{18}$ resistance arteries. The identity of EDHF remains

Table 2 The influence of normal pressure glaucoma (NPG) on maximum relaxation (\%) and sensitivity $\left(-\log \mid C_{50}\right)$ of acetylcholine mediated relaxation in human resistance arteries

\begin{tabular}{llllll}
\hline & \multicolumn{2}{l}{ Control } & & & NPG \\
\cline { 2 - 3 } \cline { 5 - 6 } \cline { 5 - 6 } & Emax & $-\log \mid C_{50}$ & & & \\
\hline Control & $95.5(2.0)(7)$ & $7.34(0.12)(7)$ & & $93.4(2.0)(7)$ & $7.70(0.11)(7)^{*}$ \\
L-NOARG/Indo & $74.3(3.5)(5)$ & $6.91(0.11)(5)$ & & $72.0(5.1)(6)$ & $6.84(0.15)(6)$ \\
ODQ & $71.3(7.6)(5)$ & $6.98(0.27)(5)$ & & $81.3(2.6)(4)$ & $7.15(0.22)(4)$ \\
ChTx/Apa & $81.8(6.0)(5)$ & $6.85(0.21)(5)$ & & $55.4(8.16)^{*}(4)$ & $6.92(0.06)(4)$ \\
NOARG /Indo/ChTx/Ap & $15.9(5.0)(4)$ & $-\dagger$ & & $0.7(0.4)(4)$ & $-\dagger(4)$ \\
\hline
\end{tabular}

Values are expressed as mean (SEM) for arteries from (n) patients. ${ }^{*} p<0.05$ compared with arteries from control subjects, using Student's unpaired $t$ test. $\dagger-\log \mid C_{50}$ not measurable because of to small relaxation. 

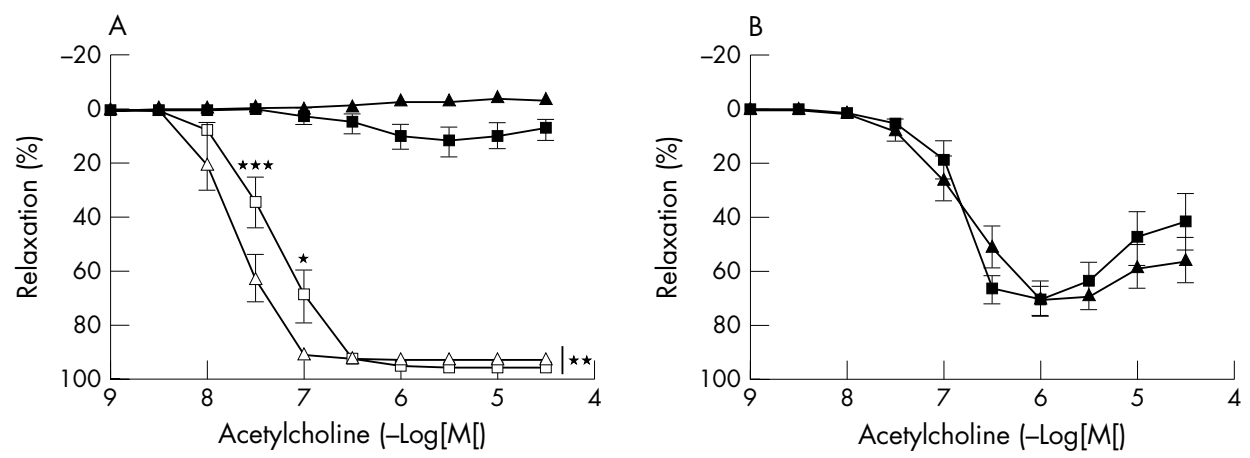

Figure 2 Concentration-response curves to acetylcholine in subcutaneous resistance arteries from patients with normal pressure glaucoma (triangles) or from matched controls (squares) in the presence of (A) without inhibitors (open symbols) or the combination of $\mathrm{N}^{\mathrm{G}}$-nitro-L-arginine, indomethacin, charybdotoxin, and apamin (filled symbols), (B) $N^{G}$. nitro-L-arginine plus indomethacin, (C) $O D Q$, or (D) the combination of charybdotoxin plus apamin. Each point represents mean (SEM).
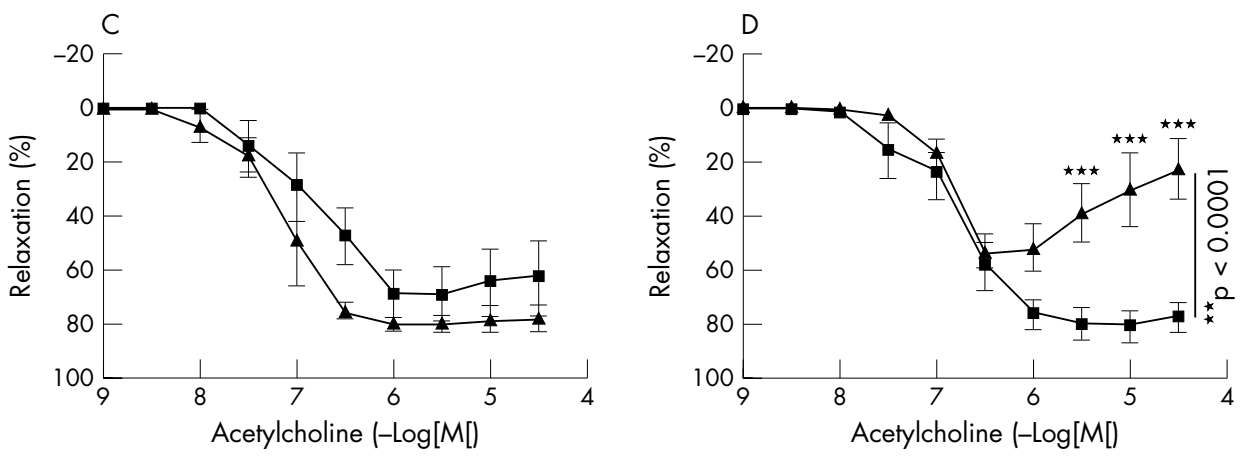

controversial but its action is now generally attributed to $\mathrm{K}^{+}$ ions, epoxyeicosatrienoic acids (EETs), and gap junctions. ${ }^{19}$ Most recent evidence supports the concept that EDHF mediated relaxation begins with an increase of calcium within the endothelial cells, leading to activation of small $\left(\mathrm{SK}_{\mathrm{Ca}}\right)$ and intermediate $\left(\mathrm{IK}_{\mathrm{Ca}}\right)$ calcium activated potassium channels and (possibly EET mediated) hyperpolarisation of the endothelial cells. Hyperpolarisation then spreads to the smooth muscle cells possibly via gap junctions and/or by the efflux of $\mathrm{K}^{+}$through the endothelial $\mathrm{SK}_{\mathrm{Ca}}$ and $\mathrm{IK}_{\mathrm{Ca}}$ channels. ${ }^{19}$

Given the inherent difficulties in obtaining human ocular arteries for in vitro functional analysis, porcine ciliary arteries were used to investigate the contribution of EDHF to relaxation in the ocular circulation. The combination of NOS and COX inhibitors with ChTx and Apa is now widely used to assess EDHF mediated relaxation. The NOS/Indo insensitive, ChTx/Apa sensitive component of relaxation is taken to be mediated by EDHF, and can also be inhibited by raising extracellular $\mathrm{K}^{+}$concentrations. ${ }^{19}$ Previous studies have suggested a role both for potassium channels and for
EDHF in relaxation of these arteries, ${ }^{15} 2021$ although the identity of the EDHF remains elusive. Our results are broadly consistent with these investigations (although methodological differences prevent direct comparison). The reduction, but not abolition, of BK mediated relaxation in the porcine vessels following inhibition of NOS activity indicated that NO was not the sole mediator of this response. It is unlikely that incomplete inhibition of NOS accounts for the residual relaxation as, in a variety of arteries, increasing inhibitor concentration $^{22}$ or using a combination of NOS inhibitor ${ }^{23}$ had no further effect. COX inhibition (with meclofenamic acid) had no further effect, supporting previous evidence that prostaglandins do not contribute to relaxation in these vessels. ${ }^{20}$ Residual relaxation was abolished, however, by the combination of $\mathrm{SK}_{\mathrm{Ca}}$ and $\mathrm{IK}_{\mathrm{Ca}}$ blockers (apamin and charybdotoxin, respectively). This supports the suggestion that EDHF makes a significant contribution to relaxation of the porcine ciliary artery. ${ }^{20} 21$ The demonstration that endothelium dependent relaxation is mediated by more than one endothelium derived relaxing factor may have significant implications for the response to disease, as the loss of a single

Table 3 The contribution of endothelium derived relaxing factors to bradykinin mediated relaxation of porcine ciliary arteries

\begin{tabular}{llll}
\hline Protocol & Treatment & $\operatorname{Emax}(\%)(\mathbf{n})$ & $-\log \mathbf{C}_{50}(\mathbf{n})$ \\
\hline (a) & Pre-incubation & $88 . .9(3.2)(14)$ & $7.68(0.19)(14)$ \\
& Control (vehicle) & $82.6(3.1)(10)$ & $7.14(0.18)(10)$ \\
& L-NAME & $53.6(12.9)^{* *}(7)$ & $7.58(0.39)(7)$ \\
(b) & Pre-incubation & $81.1(7.1)(7)$ & $7.49(0.16)(7)$ \\
& Control (L-NAME) & $45.3(10.3)(7)$ & $7.41(0.54)(7)$ \\
& L-NAME/Meclo & $47.7(12.8)(7)$ & $7.35(0.28)(7)$ \\
(c) & Pre-incubation & $81 . .3(2.9)(7)$ & $7.12(0.25)(7)$ \\
& Control (L-NAME/Meclo) & $54.3(11.7)^{*}(7)$ & $6.25(0.15)(7)$ \\
& L-NAME/Meclo/ChTx/Apa & $15.2(4.2)(7)$ & $7.16(0.39)(7)$ \\
\hline
\end{tabular}

Values are expressed as mean (SEM) for arteries from $(n)$ individuals. Data from vessels incubated with inhibitors were compared with the appropriate time dependent control using Student's unpaired $t$ test. Statistical comparison with the pre-incubation control was not made. ${ }^{*} p<0.05$ and ${ }^{* *} p<0.02$ compared with appropriate control. Emax maximum relaxation (\%); L-NAME, L-N $\mathrm{N}^{\mathrm{G}}$-nitro-arginine methyl ester; Meclo, meclofenamic acid; ChTx, charybdotoxin; Apa, apamin. 

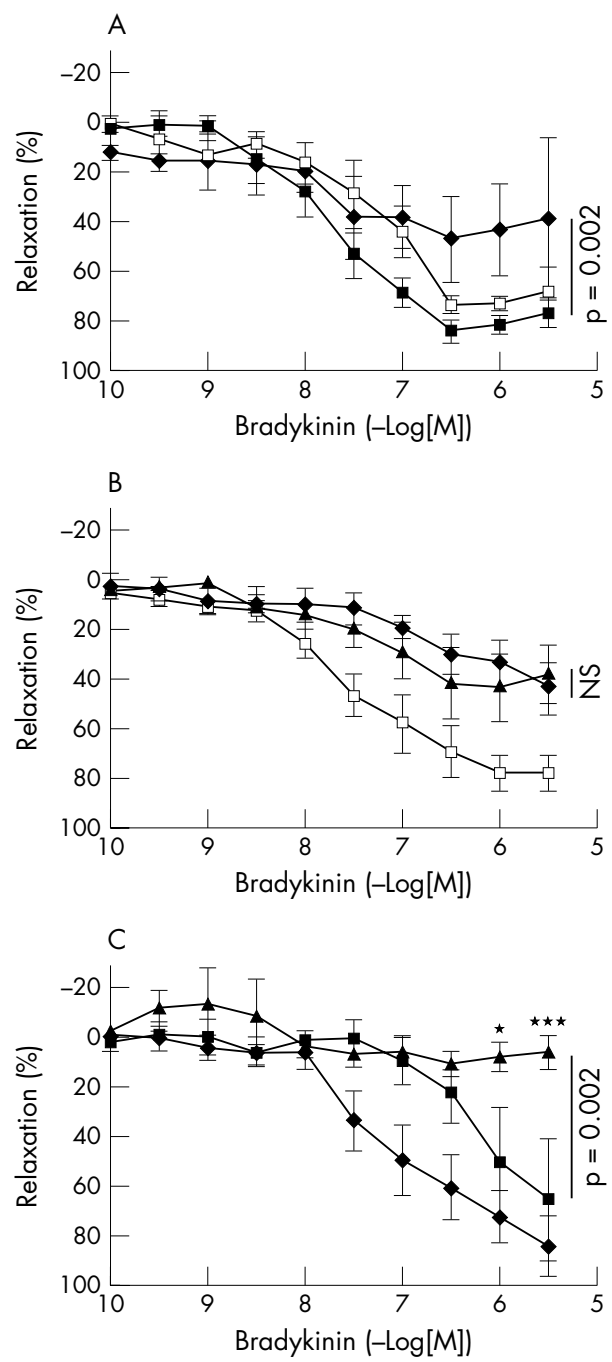

Figure 3 The influence of endothelium derived relaxing factor inhibitors on concentration response curves to bradykinin in porcine posterior ciliary arteries. (A) NO inhibition; response before (open squares; $n=14$ ) and after exposure to vehicle (solid squares; $n=10$ ) or LNAME (solid circles; $n=7$ ), (B) NO and COX inhibition responses before (open squares; $n=7$ ) and after exposure to LNAME (solid circles; $n=7$ ) or LNAME plus meclofenamic acid (solid triangles; $n=7$ ), (C) NO, COX, and EDHF inhibition; responses before (solid squares; $n=7$ ) and after exposure to LNAME plus meclofenamic acid (solid triangles; $n=7$ ) or LNAME plus meclofenamic acid, charybdotoxin, and apamin (upside down solid triangles; $n=7$ ). Each point represents mean (SEM). NO, nitric oxide; COX, cyclo-oxygenase; EDHF, endothelium derived hyperpolarising factor.

factor may not greatly affect the overall ability of the artery to relax. $^{12}$

The influence of EDHF on vascular function in disease may explain our previous demonstration that ACh mediated relaxation was not impaired in systemic (gluteal) resistance arteries from patients with NPG. ${ }^{10}$ ACh mediated relaxation of gluteal resistance arteries is mediated by both $\mathrm{NO}$ and EDHF, but not by dilator prostaglandins. ${ }^{12425}$ The current study is consistent with our previous work, with ACh mediated relaxation enhanced, rather than impaired, in arteries from patients. Complete abolition of ACh mediated relaxation using all four inhibitors together indicates that no factors other than NO, PGs, and EDHF contribute to this response. Inhibition of NOS and COX using the combination of L-NOARG and meclofenamic acid suggested that relaxa- tion caused by NO was unaltered in patients with NPG. A similar pattern was observed with inhibition of NO mediated relaxation using the soluble guanylate cyclase inhibitor (ODQ). However, in this part of the study a trend towards greater relaxation in the NPG group of vessels exposed to ODQ (although not achieving statistical significance $(p=0.3))$ suggested that NO mediated relaxation was impaired in arteries from patients. This would be consistent with our in vivo study of human forearm blood flow, using venous occlusion plethysmography. ${ }^{9}$ The similarity of relaxation with the combination of NOS and COX inhibitors may be influenced by the development of a contribution from dilator prostaglandins to the response of arteries in the NPG group. Inhibition of EDHF with ChTx/Apa indicated a significant enhancement of the contribution made by EDHF to ACh mediated relaxation in the NPG group. These results suggest, therefore, that while the development of NPG may produce a small impairment in the NO pathway, endothelium dependent relaxation in human systemic resistance arteries is maintained by enhanced activity of EDHF and, possibly, dilator prostaglandins. These results have similarities with those reported in rabbit renal arteries, in which the EDHF mediated component of relaxation was selectively enhanced in hypercholesterolaemic animals ${ }^{22}$ and in mesenteric arteries from diabetic rats in which EDHF mediated relaxation was attenuated ${ }^{26}$ This suggests that the balance between different endothelium derived relaxing factors may change in resistance arteries from patients with NPG, thus accounting for the maintained endothelium dependent relaxation in these vessels.

The interpretation of these results is complicated by the fact that relative contributions made by NO and EDHF to endothelium dependent relaxation are not necessarily additive. In control arteries L-NOARG plus indomethacin inhibited relaxation by $\sim 25 \%$ and charybdotoxin plus apamin produced $\sim 20 \%$ inhibition: combination of all four inhibitors, however, reduced relaxation by $\sim 85 \%$. This discrepancy may be the result of the inter-relation between $\mathrm{NO}$ and EDHF activities: for example, there is evidence that EDHF can influence eNOS activity. ${ }^{27}$ Thus, the increased EDHF activity may be a feedback response to reduced NO activity but there is also the possibility of a role for circulating factors, such as ascorbate, ${ }^{28}$ in the regulation of EDHF. Certainly, altered EDHF and NO activity in the present investigation cannot be attributed to hypercholesterolaemia or elevated blood pressure as these parameters were similar in patients and controls.

In vivo studies in young healthy volunteers show that NO is released continuously from human choroidal and optic nerve head vessels. ${ }^{29}$ In addition to basal NO release, the isometric exercise induced increase in choroidal blood flow is blocked by NOS inhibition..$^{30}$ More recently, it has been shown that NO contributes to basal retinal vascular tone in healthy humans and also appears to play a part in flicker induced retinal vasodilatation. ${ }^{31}$ Our previous studies have identified impaired NO mediated endothelium dependent relaxation in the systemic forearm circulation in NPG patients. ${ }^{9}$ Similar dysfunction in the ocular vasculature could lead to reduced blood flow in the retina, choroid, and optic nerve. The present study might suggest that a reduced NO mediated ocular blood flow could be compensated for by enhanced EDHF activity.

In conclusion, this study has shown that an alteration in the balance of EDRFs contributes to the maintenance of endothelium dependent relaxation in systemic resistance arteries from patients with NPG. As EDHF and NO both contribute to endothelium mediated responses in porcine ciliary arteries, similar compensation may be important in the modulating the response of the ocular circulation to disease. 


\section{ACKNOWLEDGEMENTS}

This work was supported by the Royal National Institute for the Blind (London, UK). PWFH is funded by the British Heart Foundation.

\section{Authors' affiliations}

C Cleary, C O'Brien, Department of Ophthalmology, Conway Institute, University College Dublin, Ireland

C Cleary, P McLoughlin, Department of Physiology, Conway Institute, University College Dublin, Ireland

C H Buckley, Department of Medicine, Royal Infirmary, Western

General Hospital, Edinburgh, UK

E Henry, Department of Ophthalmology, Royal Infirmary, Western

General Hospital, Edinburgh, UK

P W F Hadoke, Endocrinology Unit, Western General Hospital,

Edinburgh, UK

\section{REFERENCES}

1 Kamal D, Hitchings R. Normal tension glaucoma-a practical approach. $\mathrm{Br} J$ Ophthalmol 1998;82:835-40.

2 Goldberg I, Hollows FC, Kass MA, et al. Systemic factors in patients with lowtension glaucoma. Br J Ophthalmol 1981;65:56-62.

3 Flammer J. To what extent are vascular factors involved in the pathogenesis of glaucoma? In: Kaiser HJ, Flammer J, Hendrickson P, eds. Ocular blood flow: new insights into the pathogenesis of ocular diseases. Basle: Karger, 1996.

4 Butt Z, O'Brien C, McKillop G, et al. Color doppler imaging in untreated highand normal- pressure open-angle glaucoma. Invest Ophthalmol Vis Sci 1997;38:690-6

5 Rankin SJA, Walman BE, Buckley AR, et al. Color Doppler imaging and spectral-analysis of the optic-nerve vasculature in glaucoma. Am J Ophthalmol 1995:119:685-93.

6 Harris A, Sergott RC, Spaeth GL, et al. Color Doppler analysis of ocular vessel blood velocity in normal-tension glaucoma. Am J Ophthalmol 1994; 1 18:642-9.

7 Phelps CD, Corbett JJ. Migraine and low-tension glaucoma. A case control study. Invest Ophthalmol Vis Sci 1985;26:1105-8.

8 Drance SM, Wijsman K, Schulzer M, et al. Responses of blood flow to warm and cold in normal subjects and low tension glaucoma patients. Am J Ophthalmol 1988;105:35-9.

9 Henry E, Newby DE, Webb DJ, et al. Peripheral endothelial dysfunction in normal pressure glaucoma. Invest Ophthalmol Vis Sci 1999:40:1710-14.

10 Buckley CH, Hadoke PWF, Henry E, et al. Selective impairment of endothelium mediated inhibition of vasoconstriction in patients with normal pressure glaucoma. Br J Ophthalmol 2002;86:227-32

11 McIntyre C-A, Buckley CH, Andrews RC, et al. Endothelium-derived hyperpolarising factor and potassium use different mechanisms to induce relaxation of human sub-cutaneous resistyance arteries. Br J Pharmacol 2001;133:902-8

12 Garland CJ, Plane F, Kemp BK, et al. Endothelium-dependent hyperpolarization: a role in the control of vascular tone. Trends Pharmacol Sci 1995; 16:23-30.

13 Brandes RP, Behra A, Lebherz C, et al. N-G-nitro-L-arginine- and indomethacin-resistant endothelium-depednent relaxation in the rabbit rena artery: effect of hypercholesterolaemia. Atherosclerosis 1997;135:49-55.
14 Kagota S, Tamashiro A, Yamaguchi Y, et al. Excessive salt or cholesterol intake alters the balance among endothelium-derived factors released from renal arteries in spontaneously hypertensive rats. J Cardiovasc Pharmacol 1999:34:533-9.

15 Zhu PL, Beny JL, Flammer J, et al. Relaxation by bradykinin in porcine ciliary artery-role of nitric oxide and K+ channels. Invest Ophthalmol Vis Sci 1997;38:1761-7.

16 Mulvany MJ, Halpern W. Contractile properties of small arterial resistance vessels in spontaneously hypertensive and normotensive rats. Circ Res 1977;411:19-26.

17 Deng L-Y, Li JS, Schiffrin EL. Endothelium-dependent relaxation of small arteries from essential hypertensive patients: mechanisms and comparison with normotensive subjects and with responses of vessels from spontaneously hypertensive rats. Clin Sci 1995;88:611-22.

18 Shimokawa H, Yasutake H, Fujii K, et al. The importance of the hyperpolarising mechanism increases as the vessel size decreases in endothelium-dependent relaxations in rat mesenteric circulation. J Cardiovasc Pharmacol 1996;28:703-11.

19 Busse R, Edwards G, Feletou M, et al. EDHF: bringing the concepts together. Trends Pharmacol Sci 2002;23:374-80.

20 McNeish AJ, Wilson WS, Martin W. Dominant role of an endotheliumderived hyperpolarising factor (EDHF)-like vasodilator in the ciliary bed of the bovine isolated perfused eye. Br J Pharmacol 2001;134:912.

21 Dettmann ES, Vysniauskiene I, Wu R, et al. Adrenomedullin-induced endothelium-dependent relaxation in porcine ciliary arteries. Invest Ophthalmol Vis Sci 2003;44:3961-6.

22 Brandes RP, Behra A, Lebherz C, et al. N-G-nitro-L-arginine- and indomethacin-resistant endothelium-dependent relaxation in the rabbit renal artery: effect of hypercholesterolaemia. Atherosclerosis 1997:135:49-55

23 Plane F, Holland $M$, Waldron $C J$, et al. Evidence that anandamide and EDHF act via different mechanisms in rat isolated mesenteric arteries. Br J Pharmacol 1996;121:1509-11.

24 Urakami-Harasawa L, Shimokawa H, Nakashima M, et al. Importance of endothelium-derived hyperpolarising factor in human arteries. J Clin Invest 1997; 100:2793-9.

25 Coats $\mathbf{P}$, Johnston F, MacDonald J, et al. Endothelium-derived hyperpolarising factor. Identification and mechanisms of action in human subcutaneous resistacne arteries. Circulation 2001;103:1702-8.

26 Matsumoto T, Kobayashi T, Kamata K. Alterations in EDHF-type relaxation and phosphodiesterase activity in mesenteric arteries from diabetic rats. Am J Physiol 2003;285:H283-91.

27 Wang $H$, Lin L, Jiang JW, et al. Up-regulation of endothelial nitric oxide synthase by endothelium-derived hyperpolarizing factor involves mitogen activated protein kinase $\mathrm{C}$ signalling pathways. J Pharmacol Exp Ther 2003;307:753-64

28 McNeish AJ, Wilson WS, Martin W. Ascorbate blocks endothelium-derived hyperpolarizing fcator (EDHF)-mediated vasodilation in the bovine ciliary vascular bed and mesentery. Br J Pharmacol 2002;135:1801-9.

29 Luksch A, Polak K, Beier C, et al. Effects of systemic NO synthase inhibtion on choroidal and optic nerve head blood flow in healthy subjects. Invest Ophthalmol Vis Sci 2000;41:3080-4.

30 Luksch A, Polska E, Imhof A, et al. Role of NO in choroidal blood flow regulation during isometric exercise in healthy humans. Invest Ophthalmol Vis Sci 2003;44:734-9.

31 Dorner GT, Garhofer G, Kiss B, et al. Nitric oxide regulates retinal vascular tone in humans. Am J Physiol 2003;285:H631-6. 